\title{
YBX1 Promotes the Inclusion of RUNX2 Alternative Exon 5 in Dental Pulp Stem Cells
}

\author{
Jiaoxiang Shen ${ }^{1,2, *}$, Wenting She ${ }^{1,3, *}$, Fengxia Zhang ${ }^{1}$, Jihua Guo ${ }^{1,4}$, Rong Jia ${ }^{1}$ \\ ${ }^{1}$ The State Key Laboratory Breeding Base of Basic Science of Stomatology (Hubei-MOST) E Key Laboratory of Oral Biomedicine \\ Ministry of Education, School \& Hospital of Stomatology, Wuhan University, Wuhan, China \\ ${ }^{2}$ Department of Orthodontics, Stomatological Hospital of Xiamen Medical College, Xiamen, China \\ ${ }^{3}$ Department of Stomatology, Renmin Hospital of Wuhan University, Wuhan University, Wuhan, China \\ ${ }^{4}$ Department of Endodontics, School \& Hospital of Stomatology, Wuhan University, Wuhan, China
}

Background and Objectives: RUNX2 plays an essential role during the odontoblast differentiation of dental pulp stem cells (DPSCs). RUNX2 Exon 5 is an alternative exon and essential for RUNX2 transcriptional activity. This study aimed to investigate the regulatory mechanisms of RUNX2 exon 5 alternative splicing in human DPSCs.

Methods and Results: The regulatory motifs of RUNX2 exon 5 were analyzed using the online SpliceAid program. The alternative splicing of RUNX2 exon 5 in DPSCs during mineralization-induced differentiation was analyzed by RT-PCR. To explore the effect of splicing factor YBX1 on exon 5 alternative splicing, gaining or losing function of YBX1 was performed by transfection of YBX1 overexpression plasmid or anti-YBX1 siRNA in DPSCs. Human RUNX2 exon 5 is evolutionarily conserved and alternatively spliced in DPSCs. There are three potential YBX1 binding motifs in RUNX2 exon 5. The inclusion of RUNX2 exon 5 and YBX1 expression level increased significantly during mineralization-induced differentiation in DPSCs. Overexpression of YBX1 significantly increased the inclusion of RUNX2 exon 5 in DPSCs. In contrast, silence of YBX1 significantly reduced the inclusion of exon 5 and the corresponding RUNX2 protein expression level. Knockdown of YBX1 reduced the expression of alkaline phosphatase (ALP) and osteocalcin (OC) and the mineralization ability of DPSCs, while overexpression of YBX1 increased the expression of ALP and OC and the mineralization ability of DPSCs.

Conclusions: Human RUNX2 exon 5 is conserved evolutionarily and alternatively spliced in DPSCs. Splicing factor YBX1 promotes the inclusion of RUNX2 exon 5 and improves the mineralization ability of DPSCs.

Keywords: RUNX2, Exon 5, YBX1, Dental pulp stem cells

Received: February 21, 2021, Revised: September 3, 2021, Accepted: October 25, 2021, Published online: December 31, 2021

Correspondence to Jihua Guo

The State Key Laboratory Breeding Base of Basic Science of Stomatology (Hubei-MOST) \& Key Laboratory of Oral Biomedicine Ministry of Education, School \& Hospital of Stomatology, Wuhan University, 237 Luoyu Road, Wuhan 430079, China

Tel: +86-27-87686208, Fax: +86-27-87647443, E-mail: jihuaguo@whu.edu.cn

Co-Correspondence to Rong Jia

The State Key Laboratory Breeding Base of Basic Science of Stomatology (Hubei-MOST) \& Key Laboratory of Oral Biomedicine Ministry of Education, School \& Hospital of Stomatology, Wuhan University, 237 Luoyu Road, Wuhan 430079, China

Tel: +86-27-87686268, Fax: +86-27-87647443, E-mail: jiarong@whu.edu.cn

*These authors contributed equally to this work.

() This is an open-access article distributed under the terms of the Creative Commons Attribution Non-Commercial License (http://creativecommons.org/ licenses/by-nc/4.0/), which permits unrestricted non-commercial use, distribution, and reproduction in any medium, provided the original work is properly cited. Copyright (c) 2022 by the Korean Society for Stem Cell Research 


\section{Introduction}

RUNX2 (Runt-related transcription factor 2, also named Cbfal, Aml3 and PEBP2 $\alpha \mathrm{A}$ ) is a crucial transcription factor involved in osteogenic differentiation of mesenchymal stem cells and osteoblast (1). RUNX2 is also essential for tooth formation and tooth eruption, as well as the alveolar remodeling process (2). Mutations of RUNX2 have been identified as being responsible for cleidocranial dysplasia (CCD) (3). RUNX2 gene is located on chromosome 6 and contains eight exons, and encodes a highly conserved 128-amino acid DNA-binding domain named Runt domain $(4,5)$. The Runt domain is responsible for the transcription activity, and encoded by exons 2 to 5 . Nuclear localization signal (NLS) and part of C-terminus of the Runt domain are encoded by exon 5 (5). Exon 5 and 7 of RUNX2 are alternative exons and could be included or excluded in mature RUNX2 mRNA to produce four RUNX2 isoforms: an intact isoform (Full) and three alternative splicing isoforms $(\triangle 5, \triangle 7$, and $\triangle 5 \triangle 7)$ (Fig. 1A). The $\triangle 5$ and $\triangle 5 \triangle 7$ isoforms lack part of the C-terminus of the Runt domain, can't localize in the nucleus, and lose their DNA binding activity (6). Ge et al. (7) identified a mutation on RUNX2 exon 5 (c.634T $>$ G, p.T212P) in CCD patients who displayed significantly lower osteogenic, osteoclast-inductive, and decreased expression of MMP-9 and MMP-2. Therefore, the inclusion of exon 5 is important for the function of RUNX2. The expression and transcriptional activity of RUNX2 undergo multiple layers of regulation, including epigenetic regulation by microRNAs (8), post-translational modification by enzymes (9), and activation of promoters (10). However, little is known about how RUNX2 exon 5 alternative splicing is regulated.

RUNX2 is required for the odontoblast differentiation of dental pulp stem cells (DPSCs). Knockout of RUNX2 caused the arrested tooth development at the bud stage and the lack of odontoblast and ameloblast differentiation (11). The regulatory mechanisms of RUNX2 exon 5 alternative splicing in the differentiation of dental pulp stem cells (DPSCs) remain unknown.

In the present study, we analyzed the conservation of human RUNX2 exon 5 in mouse and rat Runx2 gene, and the alternative splicing of RUNX2 exon 5 during the dif-
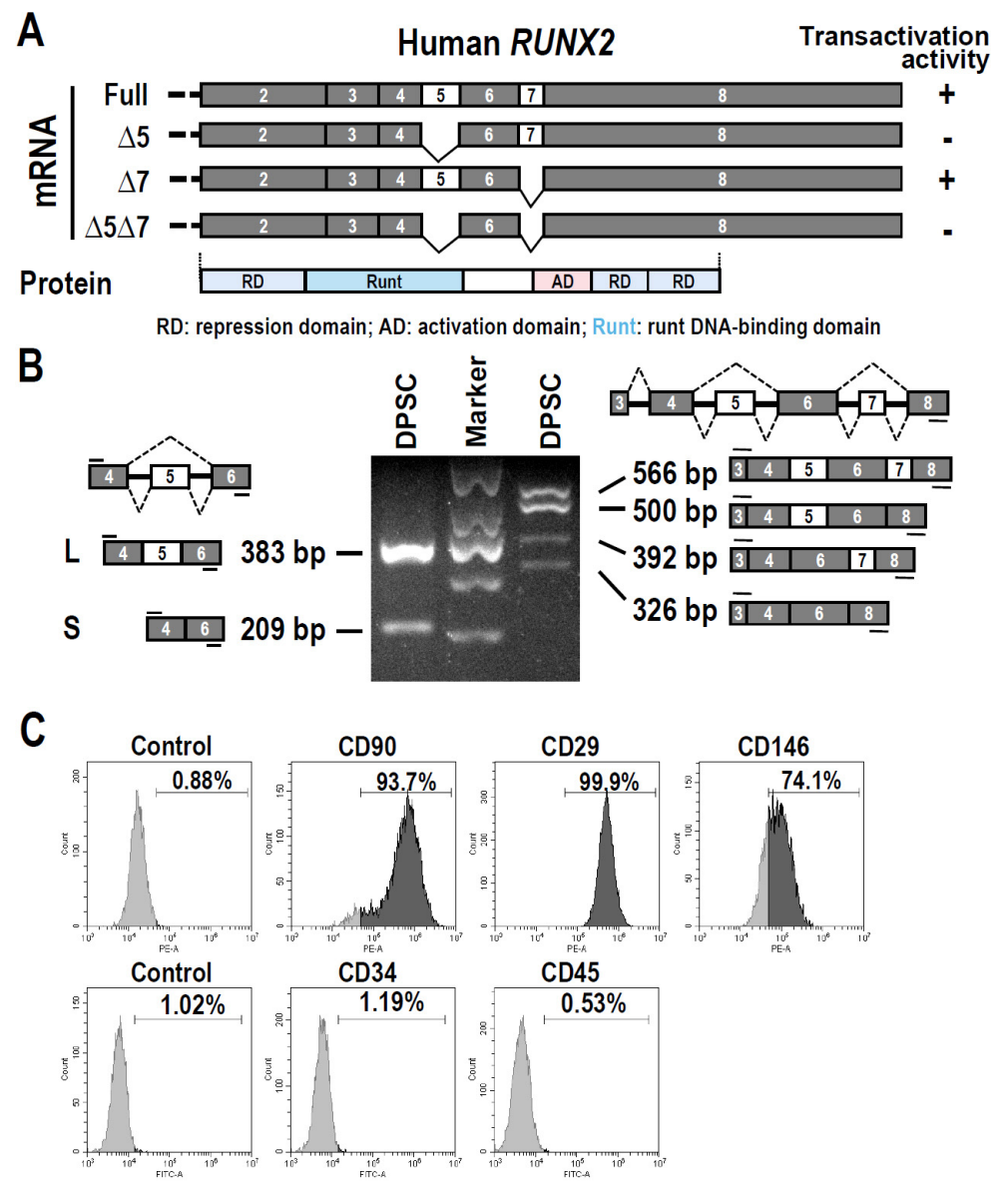

Fig. 1. Alternative splicing of RUNX2 mRNA in dental pulp stem cells (DPSC). (A) Schematic diagram of isoforms of human RUNX2. Exon 5 and 7 are two alternative exons. (B) Analysis of alternative splicing of RUNX2 mRNA in DPSCs by RTPCR. GAPDH served as a loading control. Diagrams on the left and right show the structure and size of RUNX2 pre-mRNA and spliced products, and the location of primers (short lines above or below exons). The sizes of marker bands from the top to the bottom are $700 \mathrm{bp}, 500$ bp, $400 \mathrm{bp}, 300 \mathrm{bp}$, and $200 \mathrm{bp}$, respectively. (C) Flow cytometric analysis of the purity of cultured dental pulp stem cells with stem cell biomarkers. CD90, CD29 and CD146 are mesenchymal stem cell markers, whereas CD34 and CD45 are hematopoietic stem cell markers. 
ferentiation of human DPSCs. We also analyzed the potential splicing factors for exon 5 alternative splicing with the online SpliceAid program, and further evaluated the regulatory functions of splicing factor YBX1 on RUNX2 exon 5 inclusion and mineralization formation.

\section{Materials and Methods}

\section{Cells and plasmids}

Human DPSCs were isolated from healthy human (age 22 26 years) third molars as previously described (12). This study was approved by the Ethics Committee at School and Hospital of Stomatology in Wuhan University. Informed consent was obtained from every participant. In brief, the pulp tissues were gently removed from the tooth chamber and canals, and then digested with collagenase (Roche, Germany) for $1 \mathrm{~h}$ at 37 degree. Single-cell suspensions were collected and cultured in Dental Pulp Stem Cell medium (Lonza, Germany). Primary DPSCs were analyzed by flow cytometry after incubation with mouse anti-human CD90, CD29, CD146, CD34, and CD45 antibodies (Biolegend, USA) for 30 minutes on a Beckman Coulter flow cytometer. HEK 293 cells were cultured in Dulbecco's modified Eagle medium (HyClone, USA) supplemented with $10 \%$ fetal bovine serum (FBS, HyClone, USA) and 1\% antibiotic-antimycotic (Gibco, USA). HEK 293 cells also express RUNX2 and have alternative splicing of RUNX2, which were used as a model cell to analyze the effects of YBX1 overexpression on alternative splicing of RUNX2 exon 5.

A human YBX1 expression plasmid was obtained from Addgene (\#19878), in which YBX1 was fused with a Myc epitope.

\section{siRNA and transfection}

The sequences of anti-YBX1 siRNAs are 5'CAGUUCAA GGCAGUAAAUAUGCA' (siYBX1-1) (13) and 5'GGUCA UCGCAACGAAGGUU3' (siYBX1-2) (14). The siRNAs were produced by GenePharma (China). DPSCs were transfected with $20 \mathrm{nM}$ siRNA with Lipofectamine 3000 (Invitrogen, USA). Non-specific siRNA (NS) was used as a control.

\section{Western blot}

Total cellular protein samples of HEK 293 cells or DPSCs were separated by $10 \%$ SDS-PAGE gel and transferred to a nitrocellulose filter (NC) membrane. Following antibodies were used to detect specific protein expression: mouse monoclonal anti-RUNX2 (Santa Cruz, USA), rabbit monoclonal anti-YBX1 (Abcam, USA), mouse anti-GAPDH
(Santa Cruz, USA), and horseradish peroxidase-labeled mouse anti- $\beta$-actin antibody (Sigma-Aldrich, USA).

\section{RT-PCR}

Total cellular RNAs were collected by total RNA miniprep kit (Axygen, USA). The cDNA was synthesized by using the Maxima H Minus Reverse Transcriptase from Thermo Fisher Scientific (USA). The following PCR reaction was performed with 2 x Taq Master Mix (Vazyme, China) using following primers: 5'-GACGAGGCAAGAGT TTCACC-3', 5'-ATGAAATGCTTGGGAACTGC-3', and 5'-GGTGGTAGAGTGGATGGACG-3' for RUNX2 exon 5 and 7 alternative splicing detection, 5'-GGGAACCCAGAA GGCACAGAC-3', 5'-GCCTGGGGTCTGTAATCTGACT C-3' for RUNX2 exon 5, 5'-CGTCCATCCACTCTACCACC3', 5'-ATGAAATGCTTGGGAACTGC-3' for RUNX2 exon 7 alternative splicing, 5'-GACAAGAAGCCCTTCACTGC3' and 5'-AGACTGCGCCTGGTAGTTGT-3' for alkaline phosphatase (ALP), 5'-CGCTACCTGTATCAATGGCTG3' and 5'-GCCAACTCGTCACAGTCCG-3' for osteocalcin (OC), 5'-GCTCAACCATAGAGAAAGCAAACG-3' and 5'CTTCGTTGCCTTTCCCAACTTC-3' for dentin sialophosphoprotein (DSPP), 5'-GAAGGTGAAGGTCGGAGTC3' and 5'-GAAGATGGTGATGGGATTTC-3' for GAPDH (Supplementary Table S1).

The accurate band intensity of RT-PCR product with local background subtraction were measured by using Quantity One software (Bio-Rad). The expression levels of a gene in each sample were normalized by GAPDH internal control. The ratio of RUNX2 exon 5 inclusion vs exclusion were calculated by dividing the band intensity of longer product by that of short product.

\section{Mineralization induction and alizarin red staining}

DPSCs had been treated with a differentiation medium for 21 days, which contained DMEM (Hyclone, USA), $10 \%$ fetal bovine serum (FBS, Gibco, USA), 1\% antibiotic-antimycotic, $50 \mu \mathrm{g} / \mathrm{ml}$ ascorbic acid, $10 \mathrm{mM} \beta$ glycerophosphate, and $10 \mathrm{nM}$ dexamethasone. To evaluate the mineralization, cells were washed with phosphate buffered saline (PBS) twice, then fixed by $4 \%$ paraformaldehyde solution for $15 \mathrm{~min}$ at room temperature. After washing with double distilled water, cells were stained with alizarin red stain solution for $20 \mathrm{~min}$, followed by washing with double distilled water. Staining was quantified by measuring the proportion of Alizarin-Red positive area by using imageJ. The alternative splicing of RUNX2 exon 5 and the expression of YBX1 were analyzed after three days of mineralization induction in DPSCs. 


\section{RNA-immunoprecipitation assay}

RNA-immunoprecipitation assay was performed by using Dynabeads Protein A immunoprecipitation kit (ThermoFisher, USA) and UV-crosslinking following the manufacturer's protocol. In brief, monolayer cultured DPSCs were treated with ultra-violet (UV) irradiation and lysated with RIPA buffer. RNAs associated with YBX1 protein were immunoprecipited with anti-YBX1 antibody (Abcam, USA) and analyzed by RT-PCR with a pair of primers amplifying RUNX2 exon 5.

\section{Statistical analysis}

All two-group statistical comparisons of means were performed with Student's t-test, or with Mann-Whitney U test whenever the data did not appear to have normal distribution.

\section{Results}

\section{Alternative splicing of RUNX2 exon 5 in dental pulp stem cells}

Firstly, we would like to understand whether RUNX2 is also alternative spliced in human DPSCs. We isolated and purified DPSCs from human third molar. These cells expressed mesenchymal stem cell markers, including CD90, CD29, and CD146, but were negative for hematopoietic stem cell markers CD34 and CD45 (Fig. 1C). RT-PCR analysis showed that RUNX2 exon 5 or/and exon 7 could be skipped, and produced four types of isoforms (Fig. 1B). In accord with previous publications, the isoforms without exon 5 showed no transactivation activity (6). Therefore, inclusion of exon 5 is essential for the function of RUNX2.

\section{Human RUNX2 exon $\mathbf{5}$ is evolutionarily conserved}

Then, we analyzed the evolutionary conservation of RUNX2 exon 5 in human, mouse, and rat. Similar to hu-
A

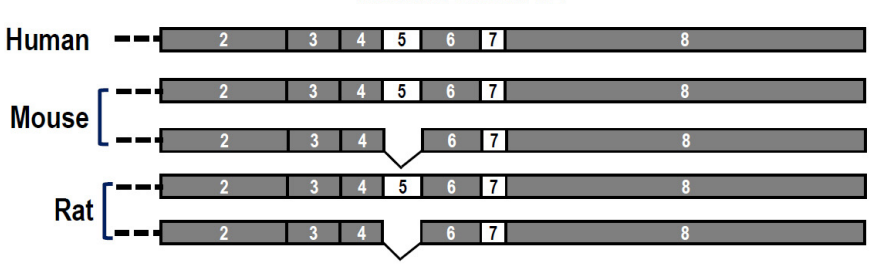

Genbank ID

NM_001024630

NM_001145920

NM_001271630

NM_001278484

B

1

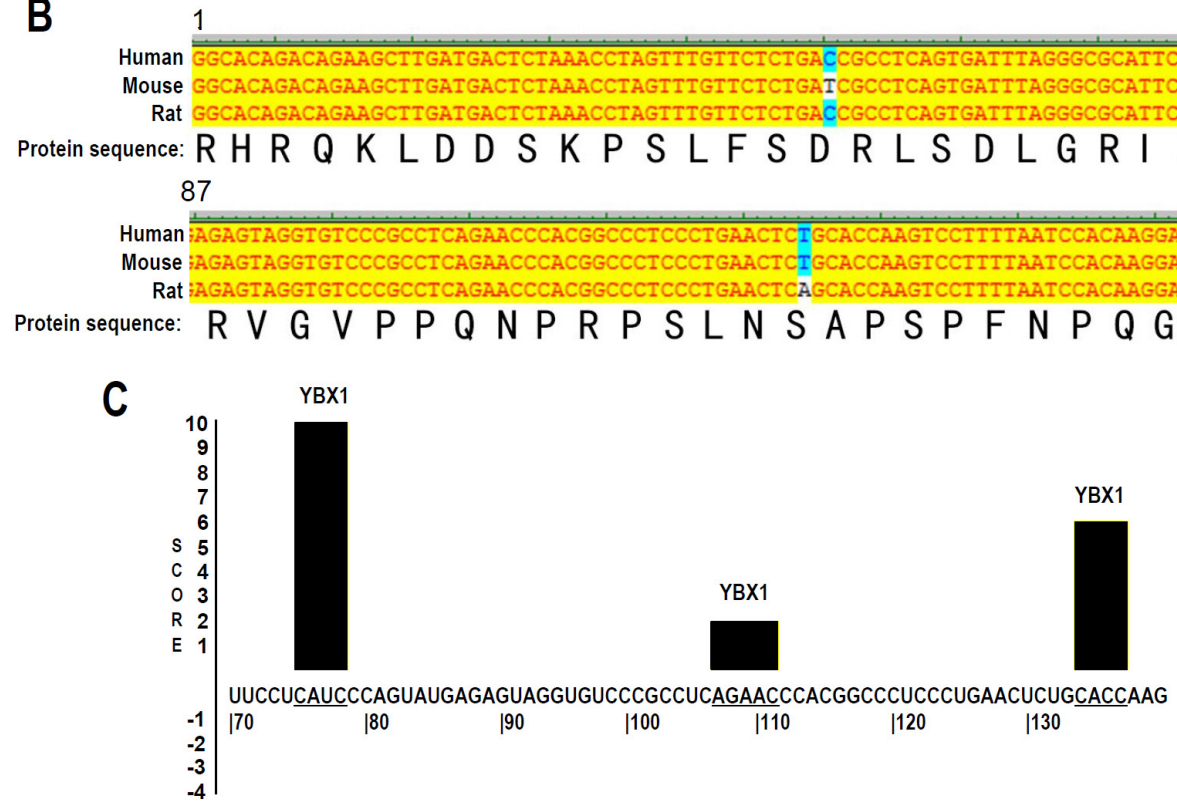

Fig. 2. Conservation of RUNX2 exon 5 in human, mouse, and rat. (A) Schematic diagram of RUNX2 exon 5 splicing in human, mouse, and rat. (B) Alignment of human RUNX2, mouse Runx2, and rat Runx2 exon 5 DNA and encoded protein sequence. (C) Predicted YBX1 binding sites with scores in human RUNX2 exon 5 RNA sequence by SpliceAid online program. Positive score represents promoting exon inclusion. 
man RUNX2, both mouse and rat Runx2 exon 5 are alternative spliced (Fig. 2A). Notably, human RUNX2 exon 5 DNA sequence is almost completely conserved in mouse and rat Runx2, except one nucleotide replacement in each species. However, the encoded protein sequences remain exactly the same (Fig. 2B). We also expanded the analyses of the evolutionary conservation of exon 5 by using UCSC genome browser, and found that the sequence of human RUNX2 exon 5 is highly conserved in rhesus, mouse, dog, elephant, chicken, partially conserved in X_tropicalis, and zebrafish (Supplementary Fig. S1). This result strongly suggested that the amino acids coded by RUNX2 exon 5 is very important, and corresponding regulatory mechanisms may be also conserved in different species. YBX1 is a splicing factor and regulates alternative splicing of mRNA by binding to $\mathrm{C} / \mathrm{A}$-rich motifs in mRNA. Interestingly, we found that there are three potential YBX1 binding motifs in RUNX2 exon 5 by using the online SpliceAid program (Fig. 2C), indicating that YBX1 may regulate RUNX2 exon 5 alternative splicing.

\section{Inclusion of RUNX2 exon 5 is enhanced during mineralization induction}

The expression of RUNX2 increased during the differentiation of DPSCs (15). However, the alternative splicing of RUNX2 exon 5 during the differentiation of DPSCs is unknown. In the present study, we induced differentiation of DPSCs with mineralization-inducing medium for 21 days. Differentiated DPSCs could form mineralized nodules and express DSPP and OC (Fig. $3 \mathrm{~A}$ and $3 \mathrm{~B}$ ). We further found that the inclusion of exon 5 significantly increased after the differentiation of DPSCs (Fig. 3C and 3D). This result is correlated with the essential role of exon 5 for RUNX2 function.

\section{YBX1 promotes RUNX2 exon 5 inclusion}

Because we found potential YBX1 binding sites in RUNX2 exon 5, we speculated that YBX1 may regulate RUNX2 exon 5 alternative splicing. We found that YBX1 expression significantly increased during the mineralization induction in DPSCs (Fig. 3E and 3F). Overexpression of YBX1 (Myc tagged YBX1) significantly increased the inclusion of RUNX2 exon 5 in both HEK 293 (Fig. 4A C) and DPSCs (Fig. 4D F). In contrast, silence of YBX1

A

B

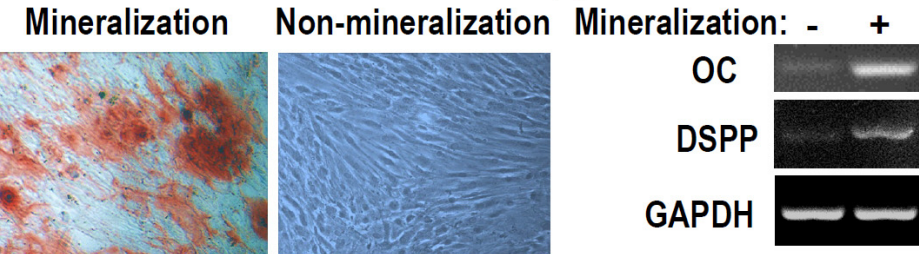

C

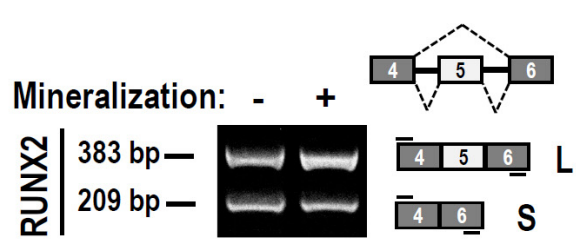

Relative L/S: 11.3 GAPDH

E

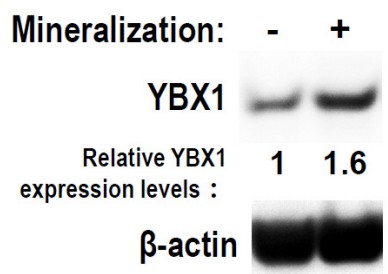

$\mathbf{F}$

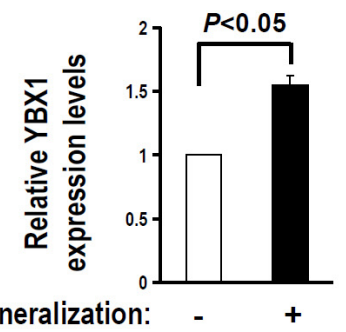

Fig. 3. RUNX2 exon 5 inclusion and YBX1 expression are enhanced after mineralization induction. (A) Alizarin red staining of DPSCs treated with or without mineralization induction for 21 days. (B, C) OC and DSPP expression (B), and alternative splicing of RUNX2 exon 5 in DPSCs after three days of mineralization induction (C) were analyzed by RT-PCR. GAPDH served as loading control. (D) Summary of four independent experiments for the ratios of inclusion versus exclusion of RUNX2 exon 5 in DPSCs after mineralization induction. $\mathrm{L}$ and $\mathrm{S}$ represent products with or without exon 5. (E) YBX1 protein expression increased after three days of mineralization induction in DPSCs. (F) Histogram showed quantification of three independent experiments for the relative levels of YBX1. Actin served as loading control. Statistical comparisons of means were performed with Student's t-test. 

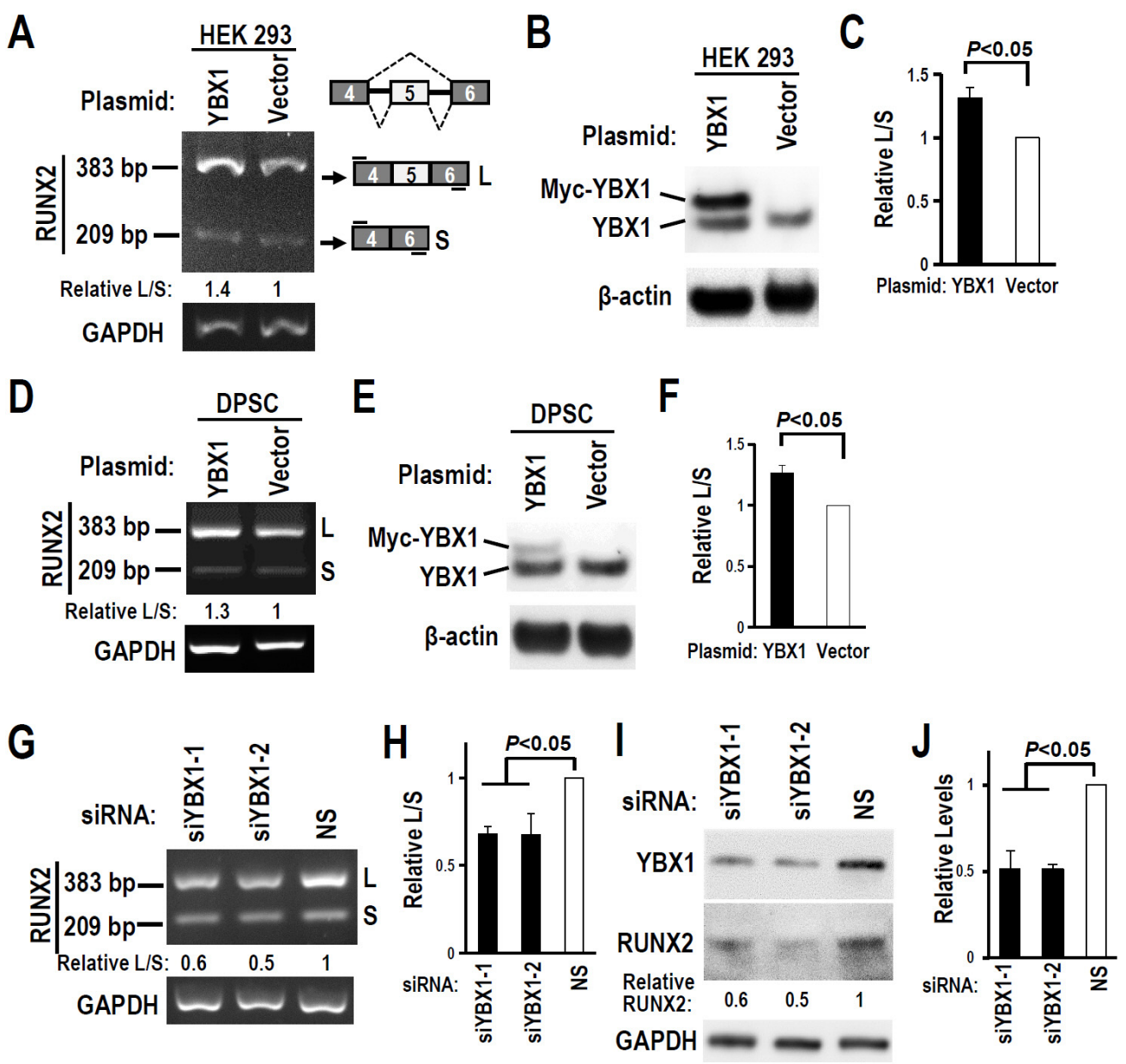

Fig. 4. YBX1 promotes RUNX2 exon 5 inclusion in DPSCs. (A $\sim$ C) Overexpression of YBX1 promoted RUNX2 exon 5 inclusion in HEK 293 cells. (D F) Overexpression of YBX1 promoted RUNX2 exon 5 inclusion in DPSCs. (B, E) Overexpression of Myc-tagged YBX1 was confirmed by Western blot. (C, F) Summary of three independent experiments for the ratios of inclusion versus exclusion of RUNX2 exon 5 in HEK 293 (C) or DPSCs (F). (G) Knockdown of YBX1 reduced RUNX2 exon 5 inclusion in DPSCs. (H) Histogram showed quantification of three independent experiments for the ratios of inclusion versus exclusion of RUNX2 exon 5 in DPSCs. (I) Knockdown efficiency of YBX1 and the expression of RUNX2 were analyzed by Western blot. GAPDH served as loading control. (J) Histogram showed quantification of three independent experiments for the expression of RUNX2 protein in DPSCs.

significantly reduced the inclusion of exon 5 (Fig. 4G and $4 \mathrm{H})$ and the corresponding RUNX2 protein expression level (Fig. 4I and 4J). These results demonstrated that YBX1 plays positive role in the inclusion of RUNX2 exon 5.

Interestingly, we also found knockdown of YBX1 increased exon 7 inclusion (Supplementary Fig. S2). However, the RUNX2 isoforms with exon 5 and without exon 7 (isoform $\triangle 7$ in Fig. 1A) maintained transactivation activity (6). YBX1 may mainly promote mineralization by increasing RUNX2 exon 5 inclusion.

\section{YBX1 contributes to the mineralization function of DPSCs}

Next, we would like to understand the function of YBX1 during the mineralization of DPSCs. We found that overexpression of YBX1 increased the expression of ALP and OC (Fig. 5A and 5B) in DPSCs. In contrast, knockdown of YBX1 reduced the expression of ALP and OC (osteocalcin) (Fig. 5E and 5F). Furthermore, we found that the alizarin red staining of YBX1 overexpression DPSCs was stronger than that of control cells after 21 days of mineralization induction (Fig. 5C and 5D). In contrast, the alizarin red staining of YBX1 knockdown DPSCs was lower than that of control cells after 21 days of mineralization induction (Fig. 5G and 5H). These results demon- 

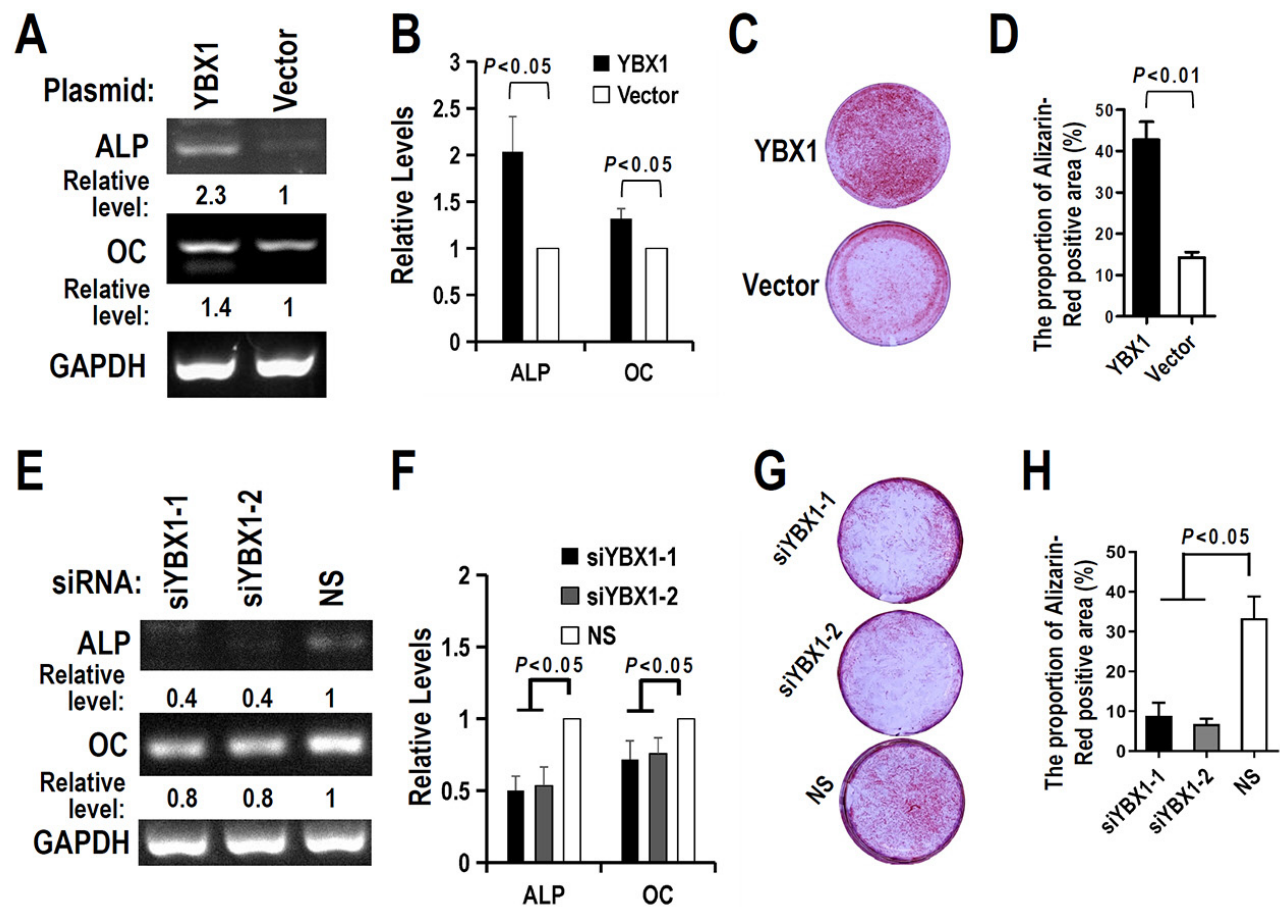

Fig. 5. YBX1 promotes the mineralization ability of DPSCs. (A $\sim D)$ Overexpression of YBX1 increased ALP and OC expression (A), and mineral formation by DPSCs in differential medium (C). (B, D) Summary of three independent experiments for ALP and OC expression (B) or mineral formation (D) by DPSCs. $(E \sim H)$ Knockdown of YBX1 suppressed ALP and OC expression (E), and mineral formation by DPSCs in differential medium $(G)$. $(F, H)$ Summary of three independent experiments for ALP and OC expression (F) or mineral formation $(\mathrm{H})$ by DPSCs. DPSCs were transfected with siRNA or plasmid for 24 hours, and then cultured in differential medium. ALP and OC expression were analyzed in Day 5. Mineral formation was analyzed by alizarin red staining in Day 21.
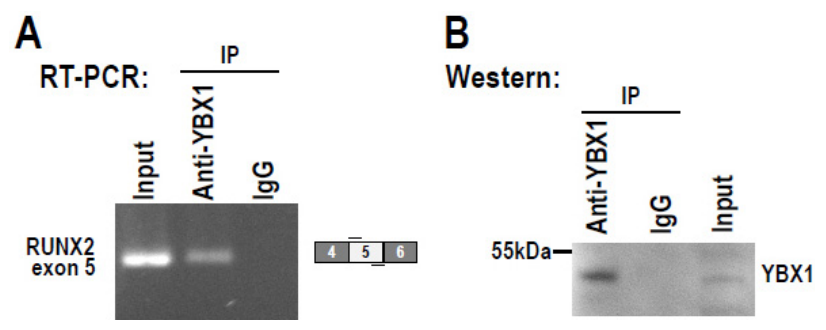

Fig. 6. YBX1 binds RUNX2 exon 5 RNA. The interaction between YBX1 and RUNX2 transcripts in DPSCs were analyzed by RNA-immunoprecipitation assay with anti-YBX1 antibody. (A) Immunoprecipitated RNA was analyzed by RT-PCR with a pair of primers amplifying RUNX2 exon 5. (B) Immunoprecipitated YBX1 protein was confirmed by Western blot.

strated that YBX1 may improve the mineralization ability of DPSCs.

\section{YBX1 binds exon 5 of RUNX2 RNA}

To further understand the regulatory mechanism of YBX1 in the alternative splicing of RUNX2 exon 5, we performed RNA-immunoprecipitation assay with anti-YBX1 antibody in DPSCs, and found that YBX1 can bind to exon 5 of RUNX2 RNA, indicating YBX1 may directly regulate the alternative splicing of RUNX2 exon 5 (Fig. 6A and 6B).

\section{Discussion}

Alternative splicing is an essential step of gene expression developed during the long-term evolution of life, which greatly enriches the coding ability of the genome, and involved in almost all biological processes, including the differentiation of DPSCs $(16,17)$. DPSCs are distributed in pulp tissue, have similar immunophenotype and pluripotent differentiation characteristics as the bone marrow mesenchymal stem cells (BMSCs). Previous studies have shown that alternative splicing plays an important role in the differentiation of DPSCs. Take Octamer-binding transcription factor 4 (OCT4) for example, it consists of three splicing variants including OCT4A, OCT4B and OCT4B1. The former mainly maintains the stemness of DPSCs, while the latter two are mainly related to stress protection and apoptosis reduction (18). Data from this study clearly indicate that during induced differentiation of DPSCs, the inclusion of RUNX2 
exon 5 increased, suggesting that alternative splicing plays an important role in differentiation of DPSCs.

Alternative splicing is regulated by splicing regulators, such as the classic SR family, hnRNP family and other RNA splicing regulators including YBX1, SAM68, TIA-1, and TIAL1 $(19,20)$. YBX1 (also known as YB-1) is a member of the Y-box binding proteins (YBX). YBX are DNA/ RNA-binding proteins with the cold shock domain, including three members (YBX1, YBX2 and YBX3), all of which can bind to Y-box sequences on DNA. YBX1 can bind to CAUC and CACC sequences on RNA and promote the splicing of exons (21). In this study, by using online SpliceAid program analysis, we found three possible YBX1 binding sites (AGAAC, CAUC and CACC) in RUNX2 exon 5, and YBX1 might promote the inclusion of exon 5. Furthermore, YBX1 can indeed promote the inclusion of RUNX2 exon 5 in DPSCs, possibly through these binding sites by directly interacting with exon 5 . YBX1 can bind to DNA and act as a transcription factor $(22,23)$. KLF4 is a transcription factor which plays a major role in the differentiation of DPSCs into odontoblasts (24). Interestingly, studies have shown that YBX1 can bind to the promoter region of KLF4 and activate KLF4 transcription with the help of other transcription factors (25), suggesting that YBX1 may be involved in the differentiation of DPSCs as a transcription factor. It should be noted that YBX1 is an oncoprotein (26). We found that overexpressing YBX1 promoted DPSCs proliferation (Supplementary Fig. S3). Further studies are required for the possible transformation effect of YBX1 in DPSCs.

RUNX2 is not only playing an essential role in osteogenesis (27), but also involved in DNA damage response, which is crucial for cancer transformation $(28,29)$. Previous study has shown that RUNX2 can interact with tumor suppressor p53, inhibit DNA damage-induced transcriptional as well as pro-apoptotic activity of p53, which is conducive to the proliferation of tumor cells (29). In addition, RUNX2 can promote the expression of MMP-2, MMP-9, MMP-13 and VEGF genes, then facilitate tumor metastasis (28). YBX1 is also closely related to the growth and metastasis of tumor cells. Schittek et al. (30) found that after inhibiting the expression of YBX1 with shRNA in metastatic melanoma cells, the growth of melanoma cells was significantly inhibited, the apoptosis of cells was significantly increased, and the expression levels of genes such as MMP-2, bcl-2, and Cyclin D1 were significantly decreased. Moreover, Homer et al. (31) found that YBX1 can also selectively change p53 activity, leading to tumor drug resistance and poor prognosis. These studies suggest that RUNX2 and YBX1 are functionally correlated. In this study, it was found that the expression level of YBX1 increased during the mineralization-induced differentiation of DPSCs, which was consistent with the increased expression of RUNX2 and the increase of the inclusion of RUNX2 exon 5, suggesting that YBX1 may play a positive regulatory role in the splicing of RUNX2 exon 5 during the differentiation of DPSCs.

There are few studies about the role of pre-mRNA alternative splicing in the differentiation of DPSCs into odontoblasts, but there are some reports on the role of pre-mRNA alternative splicing in the differentiation of osteoblasts $(32,33)$. Exon 5 of CBFB, the co-transcription factor of RUNX2, is alternatively spliced to produce two subtypes, CBFB1 and CBFB2. Jiang et al. (33) found that CBFB1 and CBFB2 played different roles in bone development, and only the deletion of CBFB2 could lead to the inhibition of osteogenic differentiation. The pre-mRNA of the transcription factor TAF4 has complex alternative splicing and can produce at least 10 isoforms. The isoforms with the deleted hTAF4-TAFH domain repress proliferation of human mesenchymal stem cells (hMSCs) and preferentially promote chondrogenic differentiation (34). However, the regulatory mechanism of alternative splicing of the pre-mRNAs of these genes is not well understood. In the present study, we initially revealed the role of RNA splicing regulator YBX1 in the regulation of RUNX2 exon 5 alternative splicing, providing a new direction for the regulatory mechanism in DPSCs differentiation.

\section{Acknowledgments}

This work was supported by Grant 81970933 from the National Natural Science Foundation of China.

\section{Potential Conflict of Interest}

The authors have no conflicting financial interest.

\section{Supplementary Materials}

Supplementary data including one table and three figures can be found with this article online at https://doi.org/10.15283/ijsc21035.

\section{References}

1. Komori T, Yagi H, Nomura S, Yamaguchi A, Sasaki K, Deguchi K, Shimizu Y, Bronson RT, Gao YH, Inada M, Sato M, Okamoto R, Kitamura Y, Yoshiki S, Kishimoto T. Targeted disruption of Cbfal results in a complete lack of bone formation owing to maturational arrest of osteoblasts. Cell 1997;89:755-764

2. Li S, Kong H, Yao N, Yu Q, Wang P, Lin Y, Wang J, 
Kuang R, Zhao X, Xu J, Zhu Q, Ni L. The role of runt-related transcription factor 2 (Runx2) in the late stage of odontoblast differentiation and dentin formation. Biochem Biophys Res Commun 2011;410:698-704

3. Cohen MM Jr. Biology of RUNX2 and cleidocranial dysplasia. J Craniofac Surg 2013;24:130-133

4. Levanon D, Groner Y. Structure and regulated expression of mammalian RUNX genes. Oncogene 2004;23:4211-4219

5. Vimalraj S, Arumugam B, Miranda PJ, Selvamurugan N. Runx2: structure, function, and phosphorylation in osteoblast differentiation. Int J Biol Macromol 2015;78:202-208

6. Makita N, Suzuki M, Asami S, Takahata R, Kohzaki D, Kobayashi S, Hakamazuka T, Hozumi N. Two of four alternatively spliced isoforms of RUNX2 control osteocalcin gene expression in human osteoblast cells. Gene 2008;413: 8-17

7. Ge J, Guo S, Fu Y, Zhou P, Zhang P, Du Y, Li M, Cheng J, Jiang H. Dental follicle cells participate in tooth eruption via the RUNX2-MiR-31-SATB2 loop. J Dent Res 2015;94:936-944

8. Narayanan A, Srinaath N, Rohini M, Selvamurugan N. Regulation of Runx2 by MicroRNAs in osteoblast differentiation. Life Sci 2019;232:116676

9. Kim HJ, Kim WJ, Ryoo HM. Post-translational regulations of transcriptional activity of RUNX2. Mol Cells 2020;43: 160-167

10. Li YL, Xiao ZS. Advances in Runx2 regulation and its isoforms. Med Hypotheses 2007;68:169-175

11. D'Souza RN, Aberg T, Gaikwad J, Cavender A, Owen M, Karsenty G, Thesleff I. Cbfal is required for epithelialmesenchymal interactions regulating tooth development in mice. Development 1999;126:2911-2920

12. Gronthos S, Mankani M, Brahim J, Robey PG, Shi S. Postnatal human dental pulp stem cells (DPSCs) in vitro and in vivo. Proc Natl Acad Sci U S A 2000;97:13625-13630

13. Kwon E, Todorova K, Wang J, Horos R, Lee KK, Neel VA, Negri GL, Sorensen PH, Lee SW, Hentze MW, Mandinova A. The RNA-binding protein YBX1 regulates epidermal progenitors at a posttranscriptional level. Nat Commun 2018;9:1734

14. Jia R, Liu X, Tao M, Kruhlak M, Guo M, Meyers C, Baker CC, Zheng ZM. Control of the papillomavirus early-to-late switch by differentially expressed SRp20. J Virol 2009;83: $167-180$

15. Camilleri S, McDonald F. Runx2 and dental development. Eur J Oral Sci 2006;114:361-373

16. Xu Y, Zhao W, Olson SD, Prabhakara KS, Zhou X. Alternative splicing links histone modifications to stem cell fate decision. Genome Biol 2018;19:133

17. Agosto LM, Lynch KW. Alternative pre-mRNA splicing switch controls hESC pluripotency and differentiation. Genes Dev 2018;32:1103-1104

18. Liu L, Huang R, Yang R, Wei X. OCT4B1 regulates the cellular stress response of human dental pulp cells with inflammation. Biomed Res Int 2017;2017:2756891

19. Busch A, Hertel KJ. Evolution of SR protein and hnRNP splicing regulatory factors. Wiley Interdiscip Rev RNA 2012;3:1-12

20. Jeong S. SR proteins: binders, regulators, and connectors of RNA. Mol Cells 2017;40:1-9

21. Wei WJ, Mu SR, Heiner $M, F u$ X, Cao LJ, Gong XF, Bindereif A, Hui J. YB-1 binds to CAUC motifs and stimulates exon inclusion by enhancing the recruitment of U2AF to weak polypyrimidine tracts. Nucleic Acids Res 2012;40:8622-8636

22. Suresh PS, Tsutsumi R, Venkatesh T. YBX1 at the crossroads of non-coding transcriptome, exosomal, and cytoplasmic granular signaling. Eur J Cell Biol 2018;97:163-167

23. Mordovkina D, Lyabin DN, Smolin EA, Sogorina EM, Ovchinnikov LP, Eliseeva I. Y-box binding proteins in $\mathrm{mRNP}$ assembly, translation, and stability control. Biomolecules 2020;10:591

24. Lin $\mathrm{H}$, Xu L, Liu H, Sun Q, Chen Z, Yuan G, Chen Z. KLF4 promotes the odontoblastic differentiation of human dental pulp cells. J Endod 2011;37:948-954

25. Shi JH, Zheng B, Chen S, Ma GY, Wen JK. Retinoic acid receptor $\alpha$ mediates all-trans-retinoic acid-induced Klf4 gene expression by regulating Klf4 promoter activity in vascular smooth muscle cells. J Biol Chem 2012;287:1079910811

26. Alkrekshi A, Wang W, Rana PS, Markovic V, Sossey-Alaoui $\mathrm{K}$. A comprehensive review of the functions of YB-1 in cancer stemness, metastasis and drug resistance. Cell Signal 2021;85:110073

27. Pratap J, Galindo M, Zaidi SK, Vradii D, Bhat BM, Robinson JA, Choi JY, Komori T, Stein JL, Lian JB, Stein GS, van Wijnen AJ. Cell growth regulatory role of Runx2 during proliferative expansion of preosteoblasts. Cancer Res 2003;63:5357-5362

28. Wysokinski D, Blasiak J, Pawlowska E. Role of RUNX2 in breast carcinogenesis. Int J Mol Sci 2015;16:20969-20993

29. Ozaki T, Wu D, Sugimoto H, Nagase H, Nakagawara A. Runt-related transcription factor 2 (RUNX2) inhibits p53dependent apoptosis through the collaboration with HDAC6 in response to DNA damage. Cell Death Dis 2013; 4:e610

30. Schittek B, Psenner K, Sauer B, Meier F, Iftner T, Garbe C. The increased expression of $\mathrm{Y}$ box-binding protein 1 in melanoma stimulates proliferation and tumor invasion, antagonizes apoptosis and enhances chemoresistance. Int $\mathrm{J}$ Cancer 2007;120:2110-2118

31. Homer C, Knight DA, Hananeia L, Sheard P, Risk J, Lasham A, Royds JA, Braithwaite AW. Y-box factor YB1 controls p53 apoptotic function. Oncogene 2005;24:83148325

32. Oren A, Toporik A, Biton S, Almogy N, Eshel D, Bernstein J, Savitsky K, Rotman G. hCHL2, a novel chordin-related gene, displays differential expression and complex alternative splicing in human tissues and during myoblast and osteoblast maturation. Gene 2004;331:17-31

33. Jiang Q, Qin X, Kawane T, Komori H, Matsuo Y, Taniuchi I, Ito K, Izumi S, Komori T. Cbfb2 isoform dominates more 
potent Cbfb1 and is required for skeletal development. J Bone Miner Res 2016;31:1391-1404

34. Kazantseva J, Kivil A, Tints K, Kazantseva A, Neuman T, Palm K. Alternative splicing targeting the hTAF4-TAFH domain of TAF4 represses proliferation and accelerates chondrogenic differentiation of human mesenchymal stem cells. PLoS One 2013;8:e74799 